\title{
Eggshell Membrane as a Bioactive Agent in Polymeric Nanotopographic Scaffolds for Enhanced Bone Regeneration
}

\author{
Daum Kim ${ }^{1}$, Yonghyun Gwon ${ }^{1}$, Sunho Park ${ }^{1}$, Sujin Kim¹, Woochan Kim ${ }^{1}$, Kwidug Yun ${ }^{1}$, \\ and Jangho Kim ${ }^{1}$ \\ ${ }^{1}$ Chonnam National University
}

September 21, 2020

\begin{abstract}
A bone regeneration scaffold is typically designed as a platform to effectively heal a bone defect while preventing soft tissue infiltration. Despite the wide variety of scaffold materials currently available, such as collagen, critical problems in achieving bone regeneration remain, including a rapid absorption period and low tensile strength as well as high costs. Inspired by extracellular matrix protein and topographical cues, we developed a polycaprolactone-based scaffold for bone regeneration using a soluble eggshell membrane protein (SEP) coating and a nanotopography structure for enhancing the physical properties and bioactivity. The scaffold exhibited adequate flexibility and mechanical strength as a biomedical platform for bone regeneration. The highly aligned nanostructures and SEP coating were found to regulate and enhance cell morphology, adhesion, proliferation, and differentiation in vitro. In a calvarial bone defect mouse model, the scaffolds coated with SEP applied to the defect site promoted bone regeneration along the direction of the nanotopography in vivo. These findings demonstrate that bone-inspired nanostructures and SEP coatings have high potential to be applicable in the design and manipulation of scaffolds for bone regeneration.
\end{abstract}

Eggshell Membrane as a Bioactive Agent in Polymeric Nanotopographic Scaffolds for Enhanced Bone Regeneration

Daun Kim ${ }^{+1}$, Yonghyun Gwon ${ }^{+1}$, Sunho Park ${ }^{1}$, Sujin Kim ${ }^{1}$, Woochan Kim ${ }^{1}$, Kwidug Yun², Jangho Kim*1

${ }^{1}$ Department of Rural and Biosystems Engineering, Chonnam National University, Gwangju 61186, Republic of Korea

${ }^{2}$ Department of Prosthodontics, School of Dentistry, Chonnam National University, Gwangju, 61649, Republic of Korea.

${ }^{+}$D.K. and Y.G. contributed equally to this work.

* Corresponding authors: Correspondence should be addressed to J.K. (rain2000@jnu.ac.kr ) or Y.G. (ykd@chonnam.ac.kr )

Abstract

A bone regeneration scaffold is typically designed as a platform to effectively heal a bone defect while preventing soft tissue infiltration. Despite the wide variety of scaffold materials currently available, such as collagen, critical problems in achieving bone regeneration remain, including a rapid absorption period and low tensile strength as well as high costs. Inspired by extracellular matrix protein and topographical cues, we developed a polycaprolactone-based scaffold for bone regeneration using a soluble eggshell membrane protein (SEP) coating and a nanotopography structure for enhancing the physical properties and bioactivity. The scaffold exhibited adequate flexibility and mechanical strength as a biomedical platform for bone 
regeneration. The highly aligned nanostructures and SEP coating were found to regulate and enhance cell morphology, adhesion, proliferation, and differentiation in vitro. In a calvarial bone defect mouse model, the scaffolds coated with SEP applied to the defect site promoted bone regeneration along the direction of the nanotopography in vivo. These findings demonstrate that bone-inspired nanostructures and SEP coatings have high potential to be applicable in the design and manipulation of scaffolds for bone regeneration.

Keywords: biomimetic, eggshell membrane, nanotopography, bone regeneration, tissue engineering

\section{Introduction}

Bone defects are frequent pathological problems of orthopedic and odontology diseases worldwide (Giannoudis, Dinopoulos, \& Tsiridis, 2005). In particular, bone loss due to massive trauma can exceed the critical size, beyond which self-healing is difficult. The current gold standard for treating these critical-size defects is an autogenous bone graft. However, autogenous bone grafts suffer from several limitations, including the high cost of surgery, complicated microsurgery techniques, and limited treatment of large bone defects (H. N. Kim et al., 2013; Lichte, Pape, Pufe, Kobbe, \& Fischer, 2011; Lim, Suh, Kim, Choung, \& Chung, 2011; McMahon, Wang, Skoracki, \& Mathur, 2013; Wang, Kim, Vunjak-Novakovic, \& Kaplan, 2006). Consequently, an effective platform is required to achieve the goals of bone regeneration (BR) (J. Kim et al., 2014). Several types of scaffolds have been introduced as an effective treatment method for BR. For example, the BR membrane, which is mainly used for alveolar BR during implant in the odontology field, guides the induction of surrounding bone tissue and promotes BR while preventing soft tissue invasion (Chiapasco, Casentini, \& Zaniboni, 2008; Fujihara, Kotaki, \& Ramakrishna, 2005; H, Hammerle, \& Karring, 1998; Rakhmatia, Ayukawa, Furuhashi, \& Koyano, 2013). The general conditions required for a BR scaffold are as follows: (1) excellent biocompatibility and adhesion to tissue cells, (2) porosity to maintain the permeation of nutrient sources, (3) blocking ability to inhibit the migration of regenerative inhibitory cells, (4) space maintenance ability to provide sufficient growth space for cells, (5) excellent mechanical strength and flexibility, and (6) ease of operation (Elgali, Omar, Dahlin, \& Thomsen, 2017).

BR scaffolds can be generally classified into those based on materials with an absorption property such as collagen, and those without absorption such as high-density polytetrafluoroethylene (d-PTFE) and titanium (Kasaj et al., 2008; Rakhmatia, Ayukawa, Atsuta, Furuhashi, \& Koyano, 2015; Sheikh et al., 2017; Song, Kim, \& Kim, 2007). However, the efficiency of these scaffolds to regenerate bone defects is still limited owing to quick absorption, low mechanical strength, and secondary surgery, as mentioned above. To overcome these disadvantages, researchers have focused on scaffolds based on polymers to prepare synthetic BR scaffolds, including poly(lactide-co-glycolic acid) (PLGA), polyglycolic acid (PGA), polylactic acid (PLA), and polycaprolactone (PCL) (G. Chen et al., 2013; Gentile, Chiono, Tonda-Turo, Ferreira, \& Ciardelli, 2011; Shim et al., 2013; Shim et al., 2014; Zhang et al., 2016). Among these, PCL is widely used in the biomedical field owing to its advantageous properties such as biodegradability, biocompatibility, high rigidity, and flexibility. In addition, PCL is a common material used to manufacture US Food and Drug Administration (FDA)-approved devices and is also relatively inexpensive (Gunatillake, 2003; J. C. Middleton., 2000; D. Kim et al., 2020). Therefore, PCL is considered to be one of the ideal candidates for the development of scaffolds for BR. Nevertheless, PCL still has many limitations such as its hydrophobic property and low adhesion, which leads to low cell attachment, proliferation, and differentiation.

Eggshells have long been treated as waste and the amount of eggshell waste is increasing annually (Balaz, 2014; Park et al., 2016). However, the membrane components in eggshells have proven to be useful for tissue regeneration, and many methods have been developed to reduce eggshell waste (Farjah, Heshmatian, Karimipour, \& Saberi, 2013; Kang, Chen, Okubayashi, \& Sukigara, 2012). The eggshell membrane (ESM) is a semi-permeable membrane with a fiber structure comprising an inner and outer double layer located between the eggshell and the egg white (Meng \& Deng, 2016; Park et al., 2016). The constituents include both organic and inorganic matter, with collagen (type I, V, and X), osteopontin (OPN), fibronectin, and $\mathrm{CaCO}_{3}$ as the major components (M. K. Sah \& Rath, 2016). In addition, ESM functionally prevents the mineralization of embryos, exchanges gases, and prevents bacteria from penetrating the egg (jun Jia, Guo, Yu, \& Dauan, 2011; Park et al., 2016; M. K. Sah \& Rath, 2016). Therefore, ESM has been mainly used in the 
biomedical field for wound dressings, and has also proven to be an efficient platform for tissue regeneration (Guha Ray et al., 2018; Kang et al., 2012). However, raw ESM has limitations in various applications owing to the difficulty in controlling its size and thickness. Therefore, various processing methods have been developed to overcome this limitation. For example, Yi et al. (Feng Yi, Yu, Guo, Zhang, \& Li, 2003) produced soluble eggshell membrane protein (SEP) from raw ESM using organic solvents. The fabricated SEP showed good bioactivity (e.g., adhesion and proliferation) compared to the collagen-type scaffold. In addition, Sah et al. (Mahesh Kumar Sah \& Pramanik, 2014) demonstrated increased adhesion and proliferation of cells in vitro by conjugating SEP to a porous silk fibroin scaffold, which also showed good biocompatibility in vivo. Jia et al. (J. Jia, Duan, Yu, \& Lu, 2008) further confirmed that the proliferation and adhesion of cells were improved by immobilizing PCL nanofibers using SEP, and SEP also improved the mechanical strength. In addition, amino acid compositions have been shown to have similar effects to SEP and raw ESM, suggesting that coating of SEP may have great potential for improving the limited bioactivity of PCL owing to its biomimetic role, which can offer ideal conditions for a variety of applications (F. Yi, Guo, Zhang, Yu, \& Li, 2004).

Living cells are highly sensitive to the complex, well-defined structural extracellular matrix (ECM) environment that can regulate cell fate and function. The ECM has various topographical characteristics ranging from the microscale to nanoscale. Many ECM proteins interact with a variety of factors to regulate the complex behavior of cells. The ECM of several tissues such as the bone, nerves, and skin with a well-ordered orientation represent geometric structures that form directional structures that affect cell function (D.-H. Kim, Provenzano, Smith, \& Levchenko, 2012; H. N. Kim, Hong, Kim, Kim, \& Suh, 2012; J. Kim, H. N. Kim, K. T. Lim, Y. Kim, S. Pandey, et al., 2013; J. Kim, H. N. Kim, K. T. Lim, Y. Kim, H. Seonwoo, et al., 2013). The bones consist of collagen and hydroxyapatite, and the protein fibers of the ECM are arranged in a complex hierarchical structure with grooves at the tens of micro- to nanoscale (J. Kim et al., 2014; Liu, Luo, \& Wang, 2016). The fate and function of osteogenic cells depend on the structure and orientation of the fibers in the highly aligned ECM topography. Therefore, by mimicking the ECM structure of the bone, it would be possible to promote BR by increasing the bioactivity property.

In this study, we used the following two strategies to promote BR. First, a patch-type scaffold was coated with SEP to convert the hydrophobic property to a hydrophilic property and improve the mechanical properties. Second, inspired by the ECM of the bone, we produced nanogrooves in the scaffold to improve the bioactivity of the cells. We investigated the effects of SEP function and topographical cues on the proliferation, differentiation, and morphology of osteogenic cells. In addition, we used a calvaria bone defect mouse model to evaluate whether our newly developed SEP-coated bone-inspired platform can promote BR in vivo

\section{Materials and Methods}

Design and Fabrication of PCL Patches and SEP-Coated PCL patches. The UV-assisted polyurethane acrylate (PUA; Changsung Sheet, Korea) precursor solution was dropped onto the silicon master mold containing nanosized linear grooves $(800 \mathrm{~nm})$. The silicon master mold was then covered with $100-\mu \mathrm{m}$-thick poly(ethyleneterephthalate) (SKC, Korea) film. For curing, the master mold covered with the PUA precursor was exposed to UV light $(\lambda=310-400 \mathrm{~nm}, 40 \mathrm{~W})$ for $30 \mathrm{~s}$.

The polydimethylsiloxane (PDMS) mold for manufacturing the flat/nano structure was fabricated as follows. To fabricate the flat mold, the silicon wafer used to fabricate the PDMS (Sylgard 184 Silicon elastomer, Dow Corning, USA) mold was fixed to a Petri dish. The PDMS pre-polymer was mixed with a $10 \%$ (w/w) curing agent solution and poured onto the clean silicon wafer, which was baked at 60 for at least $4 \mathrm{~h}$. The nano mold was fabricated in the same manner as the flat mold after fixing the PUA mother mold to the Petri dish. Before curing the PDMS solution, bubbles were removed from the vacuum condition for $1 \mathrm{~h}$.

To fabricate the ESM solution, the raw ESM was peeled off from the eggshell by hand. The separated raw ESM was washed several times with distilled water, dried, and then ground in a blender for 5 min. The eggshell powder was dissolved in a hotplate with a magnetic stirrer using 3-mercaptopropionic acid (Sigma- 
Aldrich, USA), acetic acid (Daejung Chemicals \& Metals, Republic of Korea), and deionized water at $120^{\circ} \mathrm{C}$ at $1500 \mathrm{rpm}$ for 3 days. The low-pH ESM solution was adjusted to $\mathrm{pH} 5$ using sodium hydroxide $(\mathrm{NaOH}$, Sigma-Aldrich, USA); the neutralized ESM was washed three times with methanol (Daejung Chemicals \& Metals, Republic of Korea) and distilled water and then dried in a vacuum oven at $70^{\circ} \mathrm{C}$ for $2 \mathrm{~h}$. Finally, the dried ESM powder was dissolved in a magnetic stirrer using $10 \%$ acetic acid.

PCL beads (Mw: 80,000; Sigma-Aldrich, USA) were dissolved in dichloromethane (18 wt.\%) using a magnetic stirrer for 1 day. To fabricate PCL patches, the PCL solution was poured onto a clean square glass $(18 \mathrm{~mm}$ $\times 18 \mathrm{~mm}$ ) and spin-coated at $3500 \mathrm{rpm}$ for $240 \mathrm{~s}$. The glass coated with PCL was pressed at $\sim 70 \mathrm{kPa}$ with flat PDMS fabricated on a hot plate at $80^{\circ} \mathrm{C}$. After the imprinting process, fabricated flat PCL patches were cooled off at room temperature and the PDMS mold was peeled off on the PCL patches. Using the same process, the PCL flat patches were pressed at $\sim 70 \mathrm{kPa}$ with a nano PDMS fabricated on a hot plate at $80^{\circ} \mathrm{C}$. Likewise, the nano patches were cooled off at room temperature and the PDMS mold was peeled off on the PCL flat patches. The fabricated PCL flat/nano patches were dip-coated using the SEP solution for $1 \mathrm{~h}$ at room temperature. After the coating, the PCL patches were vacuum treated to remove the organic solvent overnight.

Characteristics of SEP-Coated PCL Patches. High-resolution field-emission SEM images of the flat/nano surface and SEP-coated flat/nano surface of PCL topography patches were acquired using a JSM-7500F microscope (JEOL Ltd., Japan) at a resolution of $\times 5000$ with an acceleration voltage of $15 \mathrm{kV}$. AFM images of the SEP-coated PCL topography patches were acquired using an XE-100 microscope (Park Systems, Korea).

FT-IR was performed using a Spectrum 400 system (PerkinElmer, USA) to confirm the chemical bond structures of the PCL topography patches, SEP-coated PCL topography patches, and ESM solution. The contact angles were measured by acA1300-30 $\mu \mathrm{m}$ (Basler). Water droplets were dropped onto the surface of 5.0- $\mu \mathrm{m}$ PCL and SEP-coated PCL topography patches. The contact angles were averaged by measuring three other samples using ImageJ software (NIH, Bethesda, MD, USA).

The mechanical test of strain and stress of PCL and SEP-coated PCL patches was performed using MCT1150 tensile testers (A\&D Company, Japan) at a test speed of $100 \mathrm{~mm} / \mathrm{min}$. The bone-inspired PCL and SEP-coated PCL patches were measured by applying load along the direction of the aligned nanotopography. The experiments were analyzed for 10 specimens per sample with the same interval set. The normal and shear adhesion forces of PCL and SEP-coated PCL patches to porcine rind were measured using the MCT1150 instrument at a test speed of $50 \mathrm{~mm} / \mathrm{min}$. Prior to the adhesion test, the fresh porcine rind was rinsed with deionized water. PCL patches were attached to the surface of the porcine rind and were measured under a preload of $\sim 0.5 / \mathrm{cm}^{2}$ by gradually increasing the pulling weight until the adhesion force felled off.

In Vitro Study. Adherent cells on PCL and SEP-coated PCL patches were fixed with a $4 \%$ paraformaldehyde solution (Biosesang, Korea) for 15 min and permeabilized with $0.2 \%$ Triton X-100 (Biosesang, Korea). The samples were then blocked with $3 \%$ normal goat serum (NGS; Abcam, Cambridge, MA, USA) in phosphate-buffered saline (PBS; Biosesang, Korea). The patches were incubated overnight at $4^{\circ} \mathrm{C}$ with Vinculin (Millipore, Billerica, MA, USA). Following a wash in PBS, the patches were stained with TRITCconjugated phalloidin (Millipore) for $1 \mathrm{~h}$ and then with 4, 6-diamidino-2-phenylindole (DAPI; Millipore) for $3 \mathrm{~min}$. The images of the stained cells were obtained using a laser confocal scanning microscope. Osteoblast $\mathrm{s}\left(1 \times 10^{4}\right.$ cells/samples) were seeded onto the $15-\mathrm{mm}$ circle samples and cultured for $6 \mathrm{~h}, 3$ days, and 5 days at 37 in a humidified atmosphere containing $5 \% \mathrm{CO}_{2}$. The growth medium consisted of high-glucose Dulbecco's modified Eagle's medium (Cellgro, USA) supplemented with $10 \%$ fetal bovine serum (Cellgro, USA) and $1 \%$ penicillin-streptomycin (GenDEPOT, Houston, TX, USA). Every 3 days, the medium was replaced with fresh growth medium. Quantitative analysis of proliferation on the PCL and SEP-coated PCL topography patches was performed using the WST-1 assay (Takara Bio Inc., Japan). Before adding the reagent, the patches were washed with PBS. The reaction was carried out for $4 \mathrm{~h}$, and the extracted stains were measured using an enzyme-linked immunosorbent assay reader (iMark Microplate Absorbance Reader, Bio-Rad, Hercules) at $450 \mathrm{~nm}$ to quantify cell proliferation. 
The cells $\left(2 \times 10^{4}\right.$ cells/samples) were seeded onto the 15-mm circle samples and cultured for 1 day. After cell proliferation, the culture medium was replaced with osteogenic differentiation medium (100 nM dexamethasone, $50 \mu \mathrm{m}$ ascorbic acid, and $10 \mathrm{mM} \beta$-glycerophosphate; Sigma Aldrich, USA). After 3 days, the medium was replaced with fresh osteogenic differentiation medium. After 7 and 14 days, the cells were washed with PBS and fixed in $4 \%$ paraformaldehyde for $15 \mathrm{~min}$. Alkaline phosphatase blue membrane solution (Sigma Aldrich, USA) staining was performed to confirm the early osteogenic differentiation of osteoblasts on the sample surfaces. The stained cells were de-stained with SensoLyte pNPP Alkaline Phosphatase Assay Kit (AnaSpec, Inc., USA), and the extracted stains were measured using a microplate reader at $405 \mathrm{~nm}$ to quantify the osteogenic differentiation of osteoblasts. ARS (Sigma-Aldrich, USA) staining was performed to confirm the later osteogenic differentiation of osteoblasts on the sample surfaces based on the degree of mineralization. The stained cells were de-stained with cetylpyridinium chloride (Sigma Aldrich, USA), and the extracted stains were measured using a microplate reader at $595 \mathrm{~nm}$ to quantify the osteogenic differentiation.

A thin PDMS slab was utilized to generate a cell-free area for investigating the migration and wound-healing effects of osteoblasts on the samples. Specifically, a 3-mm-thick PDMS sheet was cut into slabs with a 3 -mm round using a steel punch. The PDMS slabs were placed onto the patch surface. Osteoblasts $\left(1 \times 10^{5}\right.$ cells/samples) were cultured on the wound generation samples. The PDMS slabs were then removed manually with sharp tweezers and the cells of the wound healing system were observed at $24 \mathrm{~h}$ by immunofluorescence staining using a fluorescence microscope (Zeiss, Germany). The cell-covered area and migration speed in wound-healing analysis were investigated using ImageJ software.

For western blot analysis, RIPA Cell Lysis buffer (Biosesang, Korea) with Xpert proteinase inhibitor cocktail (GenDEPOT, Huston, TX, USA) were used to extract proteins from the cells. Lysed protein was incubated on ice for $30 \mathrm{~min}$ and then centrifuged at 13,000 rpm for $30 \mathrm{~min}$ at $4^{\circ} \mathrm{C}$. Protein concentrations were analyzed using the DC protein assay kit (Bio-Rad, Hercules, CA, USA) and the Lowry assay method. Equal amounts of proteins were loaded on sodium dodecyl sulfate-polyacrylamide gel electrophoresis gels and then transferred onto a polyvinylidene difluoride membrane (Millipore, IPVH00010) using an electroblotting apparatus at a constant voltage of $30 \mathrm{~V}$ for $1 \mathrm{~h}$. The membrane was blocked for $1 \mathrm{~h}$ with $5 \%$ skim milk powder in Tris-buffered saline with Tween (TBS-T). Subsequently, the membrane was incubated with the following primary antibodies overnight at $4^{\circ} \mathrm{C}$ : OPN (1:2000, Abcam, Cambridge, MA, USA), ERK (1:1000, Santa Cruz Biotechnology, CA, USA), FAK (1:1000, Cell Signaling Technology, Beverly, MA, USA), and GAPDH (1:5000, Abcam, Cambridge, MA, USA). Goat anti-mouse IgG (H+L)-HRP and goat anti-rabbit IgG (H+L)HRP (GenDEPOT, Houston, TX, USA) were used as the secondary antibodies at a dilution of 1:10,000 for $1 \mathrm{~h}$ at room temperature, and then the membrane was washed three times for 10 min each in TBS-T. Bands were detected using West Pico PLUS Chemiluminescent Substrate (Thermo Scientific, Waltham, MA, USA). Quantification of the western blot was performed using image lab 6.0 (Bio-Rad, Hercules, CA, USA) software with normalization to the level of the entire protein.

In Vivo Animal Study. The animal study was approved by the Ethics Committee of Chonnam National University (CNU IACUC-YB-2019-37). Twenty-two male mice (C57Bl/6N) were assigned into two groups of 11 mice each: uncoated PCL topography patches and SEP-coated PCL topography patches. The mice were fully anesthetized with an intraperitoneal injection of zoletil $(0.006 \mathrm{cc} / 10 \mathrm{~g})$ and rumpun $(0.004 \mathrm{cc} / 10 \mathrm{~g})$, and then the head was shaved and disinfected. The bones were exposed by incising the skin approximately $3.0 \mathrm{~cm}$ above the calvarial bone. A 3-mm bone defect was made on the right and left sides of the revealed calvarial bone using an electric drill. Patches were placed onto the calvarial bone defects. After suturing the skin, the ambient temperature was raised, and the mice were woken up from the anesthesia. The mice were sacrificed 3 weeks after surgery to obtain tissues including the defect region and the calvaria bone.

Calvaria bone tomography was performed with Skyscan001172 (Skyscan, Konitch, Belgium) microcomputed tomography $(\mu-\mathrm{CT})$ at a resolution of 11.38 pixels and exposure time of $316 \mathrm{~ms}$, with an energy source of $80 \mathrm{kV}$ and current of $124 \mathrm{uA}$. An average of 488 slices of calvarial bone was scanned. The $\mu$-CT images were analyzed using MIMICS 14.0 3D imaging software (Materialise's Interactive Medical Image Control 
System, Leuven, Belgium). The calvarial bone specimens were fixed in $10 \%$ formalin and decalcified in a 0.5 $\mathrm{M}$ ethylenediaminetetraacetic acid ( $\mathrm{pH}$ 7.4) solution at room temperature for 7 days. After the specimens were embedded in paraffin, they were cut into 5 - $\mathrm{mm}$-thick sections and then stained with $\mathrm{H} \& \mathrm{E}$. Images were obtained by Aperio ImagesScope (Leica, CA, USA) software.

Statistical Analysis. Student's t-tests were used for statistical analysis. All quantitative results are presented as mean \pm standard deviation.

3.Results

\subsection{Design and Characteristics of the SEP-Coated PCL Flat Patch}

Inspired by the collagen-based ESM, to improve the low bioactivity and poor mechanical properties of PCL, it was coated with SEP as a protein signal. Toward this end, we first designed PCL-based patches with a flat topography to promote BR using capillary force lithography technology and dip coating. Figure $\mathbf{1}$ a shows a schematic of the SEP-coated PCL flat patch and optical images of a fabricated patch (see the Experimental Section for the detailed design process).

Scanning electron microscopy (SEM) images of the fabricated flat patch surface showed the spotless, clean topography of the flat structure. SEP-coated flat topography surfaces were identical to the uncoated flat topography surfaces (Figure $\mathbf{1}$ b). Atomic force microscopy (AFM) observations were made to further examine the detailed morphologies the SEP-coated flat topography, demonstrating a higher-order structure than the uncoated flat topography patch. Although the height of the surface was increased by the SEP coating, the surface was irregular because of dip coating (Figure $1 \mathrm{c}$ ). The functional groups between SEP and the PCL flat topography were investigated by Fourier transform-infrared (FT-IR) spectroscopy. The absorption bands in the spectrum of PCL with a flat topography showed $\mathrm{CH}_{2}$ asymmetric stretching at 2945 $\mathrm{cm}^{-1}$, symmetric stretching at $2866 \mathrm{~cm}^{-1}, \mathrm{C}=\mathrm{O}$ stretching vibration at $1721 \mathrm{~cm}^{-1}$, and stretching of $\mathrm{C}-\mathrm{O}$ at $1160 \mathrm{~cm}^{-1}$. The absorption bands in the spectrum of SEP typically showed $\mathrm{O}-\mathrm{H}$ stretching at $3338 \mathrm{~cm}^{-1}$ and $\mathrm{C}=\mathrm{O}$ stretching (amine II) at $1638 \mathrm{~cm}^{-1}$. The SEP-coated PCL flat patch exhibited the characteristics of both the PCL flat topography and SEP adsorption bands (Figure $1 \mathrm{~d}$ ). The contact angles of the uncoated PCL flat patches (hereafter referred to as "flat") and SEP-coated PCL flat patches (hereafter referred to as "e-flat") were 73.20deg and 34.37deg, respectively (Figure $1 \mathrm{e}$ ), confirming the change in surface properties when PCL flat patches with hydrophobic properties were coated with SEP.

A platform for effective BR must have good mechanical properties to withstand the pressure of the soft tissue and to ensure sufficient space for new tissue (Elgali et al., 2017). Therefore, the tensile strength of the patch was measured using a tensile tester to evaluate the ability to withstand the pressure of the soft tissue following SEP coating while maintaining sufficient tissue space. The e-flat patch (14.04 MPa) showed greater mechanical stresses than the flat patch ( $10.61 \mathrm{MPa}$ (Figure $1 \mathrm{f})$. To analyze the effect of SEP on the tissue adhesion performance, we measured the normal and shear adhesion forces of the PCL patches on a porcine rind tissue surface under a preload of $\sim 0.5 / \mathrm{cm}^{2}$. As shown in Figure $1 \mathrm{~g}$, the e-flat topography displayed higher normal $\left(1.16 \mathrm{~N} / \mathrm{cm}^{2}\right)$ and shear $\left(0.046 \mathrm{~N} / \mathrm{cm}^{2}\right)$ adhesion forces compared to those of the flat topography (normal: $0.76 \mathrm{~N} / \mathrm{cm}^{2}$, shear: $0.042 \mathrm{~N} / \mathrm{cm}^{2}$ ).

\subsection{In Vitro Effects of the SEP-Coated PCL Flat Patch on Osteoblasts}

SEP is known to affect living cell bioactivity (Bae et al., 2015; F. Yi et al., 2004). Therefore, the effects of the PCL flat patches on osteoblasts were evaluated to confirm the influence of SEP on cell function (e.g., cell shape and focal adhesion) at the single-cell level by immunostaining. As shown in Figure 2a, SEP greatly influenced the osteoblasts as evidenced by the extensive cytoskeletal structure on the substrate. The single cells on a flat topography showed a random shape and orientation. The cells on the e-flat topography showed a wider morphology than those on the flat topography. The cell elongation factor (CEF; long axis/short axis) was measured to quantify the observed cell polarity. Surprisingly, the CEF of cells on the e-flat topography patches was reduced compared to that on the flat topography patches. We also analyzed the nuclei of the cells based on calculation of the nuclear elongation factor (NEF; long axis/short axis), since SEP has been 
reported to affect the structure and function of the nucleus in the substrate (J. Kim, H. N. Kim, K. T. Lim, Y. Kim, H. Seonwoo, et al., 2013). There were substantially more nuclei in cells on the e-flat topography than in cells on the flat topography. Therefore, the NEF of the e-flat topography was lower than that of the flat topography. We also calculated the cell shape index (CSI; cell area/cell perimeter ${ }^{2}$ ) and nucleus shape index (NSI; nucleus area/nucleus perimeter ${ }^{2}$ ) as alternative morphological indices (J. Kim, H. N. Kim, K. T. Lim, Y. Kim, H. Seonwoo, et al., 2013). The cells on the e-flat topography showed a higher CSI value than those on the flat topography, whereas the NSI of cells was lower on the e-flat topography (Figure $2 \mathrm{~b}$ ). The elongation factor and spreading area showed a positive correlation (Figure 2c), indicating that the body and nucleus of cells can interact closely to regulate their behavior. Staining of focal adhesion markers was performed to confirm cell adhesion. With SEP coating, the cells were attached to the flat substrate over a wider range (Figure 2d). Accordingly, it was confirmed that the focal adhesion clustering size and number were higher in the e-flat structure by approximately 1.6 and 3.7 times, respectively. After $6 \mathrm{~h}$, more cells adhered to the e-flat topography patch than to the flat topography patch. After 3 days of cell culture, cell proliferation was slightly higher than that observed at $6 \mathrm{~h}$ of cell culture, and quite high proliferation was observed after 5 days. There was no significant difference between the two groups according to the SEP coating, but good proliferation was observed (Figure 2e).

The ESM is composed of both organic (80-85\%) and inorganic (15-20\%) constituents, including OPN, collagen, fibronectin, and $\mathrm{CaCO}_{3}$, which could promote BR. OPN is a human bone component, collagen type I has high osteocompatibility, and fibronectin is related to the adhesion and differentiation of cells. $\mathrm{CaCO}_{3}$ facilitates bone formation (M. K. Sah \& Rath, 2016). Therefore, we conducted alkaline phosphatase (ALP; an early BR marker) and alizarin red S (ARS; a late BR marker) to confirm BR. For ALP activation, osteoblasts were cultured for 7 days in an osteogenic induction medium, and the ALP enzyme of differentiated cells was stained in each patch. Higher ALP activity was observed on the e-flat topography than on the flat topography (Figure $2 \mathrm{f}$ ). For mineralization assessment, osteoblasts were cultured for 14 days in an osteogenic induction medium. ARS staining revealed higher calcium levels on the e-flat topography than on the flat topography (Figure $2 \mathrm{~g}$ ). Our results demonstrated that SEP coating could successfully regulate cellular behavior with potential to repair tissue in vivo or to be used as a regenerative synthetic ECM.

\subsection{Design and Characteristics of the SEP-Coated PCL Nanopatch}

The results summarized above demonstrated that SEP improves the mechanical properties and bioactivity of PCL flat patches. We next attempted to mimic the nanostructure of the ECM to further improve the function (e.g., morphology and differentiation) of cells on a structural orientation. Similar to the design of the flat patch topography, using capillary force lithography technology and dip-coating, we designed a PCL-based nanotopography patch to promote BR regeneration.Figure 3 a shows a schematic of the fabrication method of flexible PCL nanostructure (hereafter "Nano") and SEP-coated flexible PCL nanostructure (hereafter "eNano") patches inspired by the bone ECM. The SEM images of the fabricated nanopatch surfaces showed a spotless, clean topography of the nanostructures with 800-nm grooves. SEP-coated nanotopography surfaces were similar to the uncoated nanotopography surfaces (Figure $3 \mathrm{~b}$ ). We performed AFM observations to further examine the detailed morphologies the SEP-coated nanotopography, which showed a higher-order structure than the uncoated nanotopography (Figure $3 \mathrm{c}$ ). The functional groups between the SEP and PCL (uncoated) nanotopography were investigated by FT-IR spectroscopy. The absorption bands in the spectrum of the PCL nanotopography showed $\mathrm{CH}_{2}$ asymmetric stretching at $2944 \mathrm{~cm}^{-1}$, symmetric stretching at $2866 \mathrm{~cm}^{-1}, \mathrm{C}=\mathrm{O}$ stretching vibration at $1721 \mathrm{~cm}^{-1}$, and stretching of $\mathrm{C}-\mathrm{O}$ at $1161 \mathrm{~cm}^{-1}$. The absorption bands in the spectrum of the SEP nanotopography typically showed $\mathrm{O}-\mathrm{H}$ stretching at $3338 \mathrm{~cm}^{-1}$ and $\mathrm{C}=\mathrm{O}$ stretching (amine II) at $1638 \mathrm{~cm}^{-1}$. The E-Nano patch showed both the PCL nanotopography and SEP adsorption band characteristics (Figure $3 \mathrm{~d}$ ). The contact angles of the Nano patches were also measured to determine the change in surface properties when coated with SEP. Nano and E-Nano patches showed contact angles of $81.72 \mathrm{deg}$ and $35.01 \mathrm{deg}$, respectively. (Figure $3 \mathrm{e}$ ).

The tensile strength of the nanopatches was measured when loaded along the aligned nanotopography direction. Interestingly, when the load was applied toward the orientation of the aligned topography, the 
nanotopography showed greater mechanical stresses than the flat topography and increased by approxi-

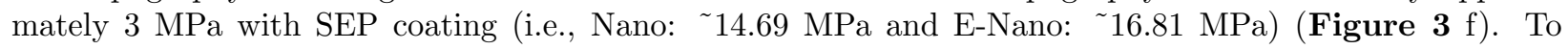
analyze the effect SEP and the nanotopography on tissue adhesion, we measured the normal and shear adhesion forces of the PCL nanopatches on a porcine rind tissue surface under a preload of $\sim 0.5 / \mathrm{cm}^{2}$. As shown in Figure $3 \mathrm{~g}$, the e-Nano patch displayed higher normal $\left(1.32 \mathrm{~N} / \mathrm{cm}^{2}\right)$ and shear $\left(0.080 \mathrm{~N} / \mathrm{cm}^{2}\right)$ adhesion forces compared with the Nano patches (normal: $1.00 \mathrm{~N} / \mathrm{cm}^{2}$, shear: $0.056 \mathrm{~N} / \mathrm{cm}^{2}$ ), likely due to the increase in the adhesion force imparted by the conjugation of PCL patches and SEP.

\subsection{In Vitro Effects of the SEP-Coated PCL Nanopatch on Osteoblasts}

A nanostructure is well known to affect cellular morphology and function, and SEP coating is known to affect cell function and behavior (J. Kim et al., 2017; J. Kim, H. N. Kim, K. T. Lim, Y. Kim, H. Seonwoo, et al., 2013; Mahesh Kumar Sah \& Pramanik, 2014; F. Yi et al., 2004). Therefore, analysis of osteoblasts on the PCL nanopatches was performed to confirm the effects of SEP and nanotopographical cues on cell function (e.g., cell shape, focal adhesion, and orientation) at the single-cell level by immunostaining. As shown in Figure 4 a, nanotopographical cues and SEP greatly influenced the cells as evidenced by the aligned cytoskeletal structure on the substrate. The single cells on the SEP coating and nanotopography showed a highly aligned shape and orientation along a unidirectionally oriented topography substrate. In addition, the cells adhered closely along the direction of the substrate. The CEF of the cells of the SEP-coated nanotopography was approximately double that of cells on the uncoated nanotopography, and the NEF was also higher in cells on the SEP-coated nanotopography than for those on the uncoated nanotopography. By contrast, the CSI and NSI were lower in cells on the SEP-coated nanotopography than for those on the uncoated nanotopography (Figure $4 \mathrm{~b}$ ). The elongation factor and spreading area showed a strong positive correlation (Figure 4 c), indicating that the body and nucleus of cells on the SEP coating and nanotopographical cues can interact closely to regulate their behavior. The cells adhered well to the SEP-coated nanostructure after $6 \mathrm{~h}$. After 3 and 5 days, the cells proliferated well (Figure 4 d). Both ALP (early) and ARS (late) staining demonstrated improved bone differentiation in the E-Nano patch (Figure 4 e andSupporting $\mathbf{3 b}$ ). We confirmed osteogenesis-related protein expression via western blotting to demonstrate BR from the nanotopographical cues and SEP. The protein expression results showed upregulation of OPN on the E-Nano patch relative to that on the Nano patch. Therefore, our results demonstrated that not only the SEP coating but also the nanostructure could successfully regulate cellular behavior and potentially repair tissue in vivo or to be used as a regenerative synthetic ECM.

\subsection{Effect of SEP Coating and Nanotopographical Cues In Vivo}

All mice used in the in vivo studies survived to the sacrifice date, and no adverse reaction was observed. SEP-coated and uncoated flat topography patches $(5 \mathrm{~mm}$ diameter) were placed on the left calvarial bone defect, and SEP-coated and uncoated nanotopography patches were placed on the right calvarial bone defect. No infection or inflammatory reaction was observed in any of the mice throughout the postoperative period. The patches remained for 3 weeks without deformation. For quantitative assessment of the nanotopography and SEP effects on bone formation, we performed micro-computed tomography $(\mathrm{CT})$ and three-dimensional (3D)-image conversion using MIMICS 14.0 software on new bone defects in vivo. As shown in the 3D images (Figure $\mathbf{5}$ a), bone formation in the PCL and SEP-coated topography patches occurred along the periphery of the bone defect and grew along the patches. At 3 weeks, the SEP-coated PCL topography patches group showed more bone formation than the uncoated PCL topography patches group at the defect. The bone volume was $0.42 \mathrm{~mm}^{3}$ in the flat topography, $0.60 \mathrm{~mm}^{3}$ in the E-flat topography, and $0.75 \mathrm{~mm}^{3}$ in the E-Nano topography groups. The bone surface was $7.8 \mathrm{~mm}^{2}$ in the flat topography, $9.39 \mathrm{~mm}^{2}$ in the E-flat topography, and $9.77 \mathrm{~mm}^{2}$ in the E-Nano topography groups (Figure $5 \mathrm{~b}$ ). This suggests that SEP-coated and nanotopography patches have potential for osteogenesis.

To confirm the BR efficacy from the SEP coating and nanotopographical cues, hematoxylin and eosin (H\&E) staining was conducted at 3 weeks after implantation. In the uncoated flat/nanotopography patch groups, bone formation was not observed, whereas in the SEP-coated flat/nanotopography patch groups, bone formation was observed at the internal space of the patch (Figure $\mathbf{5}$ a). This confirmed that more bone 
formation and a dense cytoplasm occurred on SEP-coated nanotopography structures. These results provide insight into the importance of SEP coating and nanotopographical cues for inducing bone tissue regeneration.

\section{Discussion}

Designing an effective platform that can regulate or enhance biological function is an important challenge in the tissue regeneration field. Various ECMs in the human body exist in the form of well-organized nanoscale protein fibers and are naturally oriented toward their tissue-specific functions and orientations (Bae et al., 2015; D.-H. Kim et al., 2012; H. N. Kim et al., 2013; J. Kim et al., 2014). Thus, nanotopography in the ordered structure of the ECM can regulate cell morphology and function through cell surface interactions (J. Kim, H. N. Kim, K. T. Lim, Y. Kim, H. Seonwoo, et al., 2013; Liliensiek et al., 2010; Teixeira, Abrams, Bertics, Murphy, \& Nealey, 2003). In relation to this aspect, previous studies have shown that the fate and function (i.e., proliferation, differentiation, and wound healing) of cells are sensitively controlled by establishing a variety of complex and controlled microenvironments and with appropriate stimuli (e.g., chemical, mechanical, protein, and topographical signals) in vivo(J. H. Chen \& Simmons, 2011; Das \& Zouani, 2014; H. N. Kim et al., 2013). Regarding the fabrication of platforms with topographical cues of the ECM in vivo, most studies have used structural stimuli such as hierarchical structures but were faced with technical limitations on other stimuli (J. H. Chen \& Simmons, 2011; D. H. Kim et al., 2009; D. H. Kim et al., 2010; J. Kim et al., 2017; J. Kim, H. N. Kim, K. T. Lim, Y. Kim, S. Pandey, et al., 2013). In this study, we developed a synthetic ECM with a SEP-coated nanotopography surface to achieve precise control for BR.

The ESM contains organic matter such as fibronectin and collagen, similar to the component of ECM proteins (M. K. Sah \& Rath, 2016). SEP shows a similar amino acid composition to raw ESM except for cystine and has a slightly hydrophilic property (F. Yi et al., 2004). Accordingly, many studies have been conducted using SEP as a platform for promoting cell function (J. Jia et al., 2008; G. H. Kim, Min, Park, \& Kim, 2008; Mahesh Kumar Sah \& Pramanik, 2014; F. Yi et al., 2004). Therefore, SEP protein signals affect cell function and behavior. We have presented a simple yet robust method of fabricating PCL-based nanoscale substrates using a capillary force lithography and dip-coating technique to provide these structural stimuli and protein signals. Capillary force lithography technology facilitates the design and fabrication of structural precise nano-engineered substrates. PCL was chosen for this study because it is suitable for tissue engineering and biomedical applications owing to the following features: (1) a biocompatible material used to manufacture FDA-approved devices; (2) biodegradability of approximately 2 years, making it suitable for tissues requiring long-term regeneration; and (3) robust mechanical properties and excellent flexibility. Therefore, we assert that the novel approach provides better function to osteoblasts for promoting BR, resulting in an effective platform for tissue engineering.

The functions and behaviors of cells are sensitive to topographic cues such as ECM. Our in vitro analyses demonstrated that the highly aligned nanotopographic cues affected the functions and behaviors of the cells, adjusting their morphology along the direction of the nanostructure. In addition, the SEP-coated topography regulated the morphology of the cells. The cytoskeletal morphology of the osteoblasts at the single-cell level was wider on the SEP-coated flat topography, and the cytoskeleton showed a highly aligned and oriented morphology on the SEP-coated nanotopography. Focal adhesion (FA) is as considered to be a crucial factor contributing to cellular function on a nanotopography substrate (Biggs, Richards, \& Dalby, 2010; J. Kim, H. N. Kim, K. T. Lim, Y. Kim, H. Seonwoo, et al., 2013). As shown inFigure 2 d, the SEP coating appeared to control the FA formation of cells, which would be an important modulator of cellular functions. In terms of the SEP-coated topography, flat patches showed a wider morphology because of (1) the higher number of FA clusters and (2) the thick cytoskeleton forming bunches. Nanopatches showed maximum polarity due to the highly oriented cytoskeleton bunches and FA clusters. These observations were supported by the increasing tendency of elongation of the cell body and nucleus (Figure $2 \mathrm{~b}$ and $4 \mathrm{~b}$ ). Kim et al. (J. Kim, H. N. Kim, K. T. Lim, Y. Kim, H. Seonwoo, et al., 2013) reported that the increased elongation factor of the cell was caused by a reduction in shortening. In this study, the high CEF on the SEP-coated nanotopography was due to the reduction of the short axis as in previous studies. However, the effects of the flat topography according to the long axis and short axis could not be identified owing to the widespread distribution from 
the SEP coating. Therefore, the elongation rate itself cannot provide sufficient information about the cell body except for the aspect ratio. As an alternative, CSI and NSI contain "perimeter" and "spreading area" as parameters, allowing for more reasonable quantification of cellular and nuclear morphology. Versaevel et al. (Versaevel, Grevesse, \& Gabriele, 2012) reported a quantitative model in which the cytoskeleton is regulated according to various structures and the nucleus shape is regulated accordingly; with a lower SCI, the shape becomes more bipolar with elongated cells. In this study, as the cells spread from the elongated nanotopography to the aligned orientation, the NSI decreased markedly, revealing the relationship between the cell and nucleus morphology.

Although there was no significant difference in the proliferation of osteoblasts between the uncoated PCL topographic cues and SEP-coated PCL topographic cues, we found that cell attachment and proliferation increased over time. Arias et al. (Arias et al., 2008) reported that collagen type I of ESM inhibits BR. However, collagen type I has a smaller ratio than collagen type I, and the Sah and Viana groups used the ESM in the SEP and eggshell power forms to create a platform for BR (Mahesh Kumar Sah \& Pramanik, 2014; T. Viana, 2014). Indeed, bone formation differentiation in this study was superior in the SEP-coated PCL topography patches than in the uncoated PCL topography patches based on both ALP and ARS staining. Therefore, ESM can inhibit BR, but can serve as a biomaterial to promote BR when converted to a powder and solution state.

Developing a platform to overcome the current complex surgery and enormous cost of BR is still an important challenge. A nanotopographic platform inspired by the structural features of bone tissue and the ECM has been considered a promising strategy for designing functional bones. Herein, we have proposed functional patches for BR using a biomimetic strategy based on the PCL topography and SEP coating. By mimicking the well-organized ECM of the bone tissue, our PCL matrix-based nanotopographic patches can effectively induce BR by providing an efficient environment for morphological alignment, attachment, proliferation, and differentiation of functional osteoblasts. Ourin vivo study showed that SEP coated on PCL topographical patches promoted BR. At 3 weeks, initial bone formation was increased in the SEP-coated PCL topography groups (i.e., E-flat and E-Nano) (Figure 5 a), demonstrating that SEP affects initial bone formation. Although further in-depth study is needed to precisely elucidate the underlying mechanisms, our findings demonstrate that SEP could promote early bone formation and that a nanopatterned topography resulted in superior bone formation to a flat topography. Moreover, the SEP-coated PCL topography patches provided more accurate tissue adhesion to the defected bone tissue than uncoated PCL topography patches (Figure $\mathbf{1} \mathrm{g}$ and $\mathbf{3} \mathrm{g}$ ). This finding suggests that improved patch adhesion with SEP coating on bone tissue surfaces might facilitate spontaneous BR along the nanotopography. Thus, PCL topographical patches coated with SEP might be induced to begin initial bone formation, ultimately promoting BR.

Several studies have demonstrated that a nanotopographical structure promotes cell migration. For instance, Kim et al. reported that the nanotopographical local density was able to control the wound-healing process precisely through topographical contact guidance by variable local size nanotopography. Based on these findings, we propose a possible mechanism that the nanotopography and SEP cues will synergistically promote the migration of osteoblasts. To assess the recruitment of osteoblasts and evaluate their migratory behaviors on SEP-coated PCL nanotopographical patches, we mimicked the in vivoprocess of bone repair in calvaria defects with an in vitromigration assay. We observed enhanced migratory behaviors of osteoblasts on the SEP-coated PCL nanotopographical patches compared with the nanotopographical patches. Interestingly, the cells on the E-Nano patch showed a more elongated morphology compared with those on the nano-patch (Figure $4 \mathrm{~b}$ ). In addition, the SEP coating promoted the cell migration rate. The cell migration distance $(720 \mu \mathrm{m})$ and migration velocity $(35 \mu \mathrm{m} / \mathrm{h})$ on the E-Nano patch were higher than those on the Nano patch (615 $\mu \mathrm{m}$ and $28 \mu \mathrm{m} / \mathrm{h}$, respectively). For the in vitrocell migration assay on the different surfaces, the cell-free area was determined after cell migration for 1 day (Figure 5 b). Similarly, on the SEP-nanotopographical patch, $80 \%$ of the area was covered by the migrating MG63 cells, whereas only $60 \%$ of the area was covered by cells on the nanotopographical patch. In general, wound healing is governed by two distinct cell behaviors: migration and proliferation. Thus, our results demonstrated a notable influence of the nanotopography in the wound healing process: (i) the SEP cues significantly promoted the migration of osteoblasts into the 
wound area; (ii) the orientation of the nanotopographical structure significantly affected the migration speed of the osteoblasts cells; and (iii) the orientation of the nanotopographical patch and SEP cues provided an efficient environment for constructing the structure of the native bone ECM in which osteoblasts could proliferate and migrate to the wound area.

The expression levels of ERK and FAK were examined to investigate cell function-related signaling and focal adhesion-related signaling pathways, respectively (Cheng et al., 2019; J. Kim, H. N. Kim, K. T. Lim, Y. Kim, H. Seonwoo, et al., 2013). We performed western blotting to analyze the cell-cell and cellsubstrate relationships in more detail. The extracellular-signal-related kinase (ERK) signal was higher for the E-flat group (1.03) than for the flat group (0.43), indicating greater cell-substrate interactions, and the focal adhesion kinase (FAK) signal was also greater for the E-flat (0.71) group than for the flat group (0.26), indicating greater cell-cell interactions (Figure $\mathbf{5}$ e,Supporting S3 a). Hence, we suggest that SEP cues may be able to adjust the cell-substrate interactions. Namely, the expression of ERK and FAK can be controlled by the SEP coating to induce cell body and nucleus shape changes through cell-substrate interactions (Figure $5 \mathrm{e}$ ). SEP and nanotopographical cues were found to enhance bone differentiation and mechanical properties. The SEP-coated nanotopographical patches showed improved mechanical factors, cell spreading, and osteogenesis (Figure $5 \mathrm{f}$ ).

Finally, we propose another possible application of the SEP-coated PCL matrix topography. First, a precisely defined nanotopography platform with coated SEP might allow for the fabrication of substrates by mimicking the aligned structure of other tissues (e.g., the skin, muscle, or heart). Second, SEP-coated nanotopography patches can be used in a variety of biomedical applications such as tissues that require strong support owing to their robust mechanical properties and improved adhesion to tissue bonding (Bakopoulou et al., 2019; Chamieh et al., 2016; J. Kim et al., 2014). Therefore, our SEP-coated nanotopographic patches might interact to enhance the behavior and function of the cells.

Acknowledgements

This work was supported by National Research Foundation (NRF) grants funded by the Korea Government (2016M3A9B4919374, 2019R1I1A3A0106345 and NRF-2019M3A9H1103737). This work was also supported by a grant (714002-7) from the Agricultural Robotics and Automation Research Center through the Agriculture, Food and Rural Affairs Research Center Support Program, Ministry of Agriculture, Food and Rural Affairs.

\section{References}

Arias, J. I., Gonzalez, A., Fernandez, M. S., Gonzalez, C., Saez, D., \& Arias, J. L. (2008). Eggshell membrane as a biodegradable bone regeneration inhibitor. J Tissue Eng Regen Med, 2 (4), 228-235. doi:10.1002/term. 87

Bae, W. G., Kim, J., Choung, Y. H., Chung, Y., Suh, K. Y., Pang, C., . . . Jeong, H. E. (2015). Bio-inspired configurable multiscale extracellular matrix-like structures for functional alignment and guided orientation of cells. Biomaterials, 69 , 158-164. doi:10.1016/j.biomaterials.2015.08.006

Bakopoulou, A., Georgopoulou, A., Grivas, I., Bekiari, C., Prymak, O., Loza, K., . . . Chatzinikolaidou, M. (2019). Dental pulp stem cells in chitosan/gelatin scaffolds for enhanced orofacial bone regeneration.Dent Mater, 35 (2), 310-327. doi:10.1016/j.dental.2018.11.025

Balaz, M. (2014). Eggshell membrane biomaterial as a platform for applications in materials science. Acta Biomater, 10 (9), 3827-3843. doi:10.1016/j.actbio.2014.03.020

Biggs, M. J., Richards, R. G., \& Dalby, M. J. (2010). Nanotopographical modification: a regulator of cellular function through focal adhesions. Nanomedicine, 6 (5), 619-633. doi:10.1016/j.nano.2010.01.009

Chamieh, F., Collignon, A. M., Coyac, B. R., Lesieur, J., Ribes, S., Sadoine, J., . . . Rochefort, G. Y. (2016). Accelerated craniofacial bone regeneration through dense collagen gel scaffolds seeded with dental pulp stem cells. Sci Rep, 6 , 38814. doi:10.1038/srep38814 
Chen, G., Xia, Y., Lu, X., Zhou, X., Zhang, F., \& Gu, N. (2013). Effects of surface functionalization of PLGA membranes for guided bone regeneration on proliferation and behavior of osteoblasts. $J$ Biomed Mater Res A, 101 (1), 44-53. doi:10.1002/jbm.a.34298

Chen, J. H., \& Simmons, C. A. (2011). Cell-matrix interactions in the pathobiology of calcific aortic valve disease: critical roles for matricellular, matricrine, and matrix mechanics cues. Circ Res, 108 (12), 1510-1524. doi:10.1161/CIRCRESAHA.110.234237

Cheng, Z. A., Alba-Perez, A., Gonzalez-Garcia, C., Donnelly, H., Llopis-Hernandez, V., Jayawarna, V., . . . Salmeron-Sanchez, M. (2019). Nanoscale Coatings for Ultralow Dose BMP-2-Driven Regeneration of Critical-Sized Bone Defects. Adv Sci (Weinh), 6 (2), 1800361. doi:10.1002/advs.201800361

Chiapasco, M., Casentini, P., \& Zaniboni, M. (2008). Bone Augmentation Procedures in Implant Dentistry. The International Journal of Oral \& Maxillofacial Implants, 24, 237-259.

Das, R. K., \& Zouani, O. F. (2014). A review of the effects of the cell environment physicochemical nanoarchitecture on stem cell commitment.Biomaterials, 35 (20), 5278-5293. doi:10.1016/j.biomaterials.2014.03.044

Elgali, I., Omar, O., Dahlin, C., \& Thomsen, P. (2017). Guided bone regeneration: materials and biological mechanisms revisited. Eur J Oral Sci, 125 (5), 315-337. doi:10.1111/eos.12364

Farjah, G. H., Heshmatian, B., Karimipour, M., \& Saberi, A. (2013). Using Eggshell Membrane as Nerve Guide Channels in Peripheral Nerve Regeneration. Iranian Journal of Basic Medical Sciences,, 16 (8), 901-905.

Fujihara, K., Kotaki, M., \& Ramakrishna, S. (2005). Guided bone regeneration membrane made of polycaprolactone/calcium carbonate composite nano-fibers. Biomaterials, 26 (19), 4139-4147. doi:10.1016/j.biomaterials.2004.09.014

Gentile, P., Chiono, V., Tonda-Turo, C., Ferreira, A. M., \& Ciardelli, G. (2011). Polymeric membranes for guided bone regeneration. Biotechnol J, 6 (10), 1187-1197. doi:10.1002/biot.201100294

Giannoudis, P. V., Dinopoulos, H., \& Tsiridis, E. (2005). Bone substitutes: an update. Injury, 36 Suppl 3 , S20-s27. doi:10.1016/j.injury.2005.07.029

Guha Ray, P., Pal, P., Srivas, P. K., Basak, P., Roy, S., \& Dhara, S. (2018). Surface Modification of Eggshell Membrane with Electrospun Chitosan/Polycaprolactone Nanofibers for Enhanced Dermal Wound Healing.ACS Applied Bio Materials, 1 (4), 985-998. doi:10.1021/acsabm.8b00169

Gunatillake, P. A. (2003). Biodegradable synthetic polymers for tissue engineering. European Cells and Materials, 5 , 1-16. doi:10.22203/eCM.v005a01

H, C., Hammerle, F., \& Karring, T. (1998). Guided bone regeneration at oral implant sites. Periodontology 2000, $17,151-175$.

J. C. Middleton., A. J. T. (2000). Synthetic biodegradable polymers as orthopedic devices. Biomaterials, $21,2335-2346$.

Jia, J., Duan, Y. Y., Yu, J., \& Lu, J. W. (2008). Preparation and immobilization of soluble eggshell membrane protein on the electrospun nanofibers to enhance cell adhesion and growth. $J$ Biomed Mater Res A, 86 (2), 364-373. doi:10.1002/jbm.a.31606

Jia, j., Guo, Z., Yu, J., \& Dauan, Y. (2011). A New Candidate for Guided Tissue Regeneration Biomimetic Eggshell Membrane. Iranian Journal of Medical Hypotheses and Ideas, 5 , 1-5.

Kang, J., Chen, L., Okubayashi, S., \& Sukigara, S. (2012). Preparation of electrospun polycaprolactone nanofibers with water-soluble eggshell membrane and catechin. Journal of Applied Polymer Science, 124 (S1), E83-E90. doi:10.1002/app.35538 
Kasaj, A., Reichert, C., Gotz, H., Rohrig, B., Smeets, R., \& Willershausen, B. (2008). In vitro evaluation of various bioabsorbable and nonresorbable barrier membranes for guided tissue regeneration. Head Face Med, 4 , 22. doi:10.1186/1746-160X-4-22

Kim, D.-H., Provenzano, P. P., Smith, C. L., \& Levchenko, A. (2012). Matrix nanotopography as a regulator of cell function. The Journal of Cell Biology, 197 (3), 351-360. doi:10.1083/jcb.201108062

Kim, D., Kim, W., Park, S., Kim, S., Gwon, Y., \& Kim, J. (2020). Leaf-Inspired Micro- and Nanoengineered Surfaces for Controlled Hydrophilic and Hydrophobic Properties. Macromolecular Research, 28 (1), 57-61. doi:10.1007/s13233-020-8007-2

Kim, D. H., Han, K., Gupta, K., Kwon, K. W., Suh, K. Y., \& Levchenko, A. (2009). Mechanosensitivity of fibroblast cell shape and movement to anisotropic substratum topography gradients. Biomaterials, 30 (29), 5433-5444. doi:10.1016/j.biomaterials.2009.06.042

Kim, D. H., Lipke, E. A., Kim, P., Cheong, R., Thompson, S., Delannoy, M., . . . Levchenko, A. (2010). Nanoscale cues regulate the structure and function of macroscopic cardiac tissue constructs. Proc Natl Acad Sci U S A, 107 (2), 565-570. doi:10.1073/pnas.0906504107

Kim, G. H., Min, T., Park, S. A., \& Kim, W. D. (2008). Coaxially electrospun micro/nanofibrous poly(epsilon-caprolactone)/eggshell-protein scaffold. Bioinspir Biomim, 3 , 016006. doi:10.1088/1748$3182 / 3 / 1 / 016006$

Kim, H. N., Hong, Y., Kim, M. S., Kim, S. M., \& Suh, K. Y. (2012). Effect of orientation and density of nanotopography in dermal wound healing. Biomaterials, 33 (34), 8782-8792. doi:10.1016/j.biomaterials.2012.08.038

Kim, H. N., Jiao, A., Hwang, N. S., Kim, M. S., Kang, D. H., Kim, D. H., \& Suh, K. Y. (2013). Nanotopography-guided tissue engineering and regenerative medicine. Adv Drug Deliv Rev, 65 (4), 536558. doi:10.1016/j.addr.2012.07.014

Kim, J., Bae, W. G., Choung, H. W., Lim, K. T., Seonwoo, H., Jeong, H. E., . . . Chung, J. H. (2014). Multiscale patterned transplantable stem cell patches for bone tissue regeneration. Biomaterials, 35 (33), 9058-9067. doi:10.1016/j.biomaterials.2014.07.036

Kim, J., Bae, W. G., Kim, Y. J., Seonwoo, H., Choung, H. W., Jang, K. J., . . . Chung, J. H. (2017). Directional Matrix Nanotopography with Varied Sizes for Engineering Wound Healing. Adv Healthc Mater, 6 (19). doi:10.1002/adhm.201700297

Kim, J., Kim, H. N., Lim, K. T., Kim, Y., Pandey, S., Garg, P., . . . Chung, J. H. (2013). Synergistic effects of nanotopography and co-culture with endothelial cells on osteogenesis of mesenchymal stem cells. Biomaterials, 34 (30), 7257-7268. doi:10.1016/j.biomaterials.2013.06.029

Kim, J., Kim, H. N., Lim, K. T., Kim, Y., Seonwoo, H., Park, S. H., . . . Chung, J. H. (2013). Designing nanotopographical density of extracellular matrix for controlled morphology and function of human mesenchymal stem cells. Sci Rep, 3 , 3552. doi:10.1038/srep03552

Lichte, P., Pape, H. C., Pufe, T., Kobbe, P., \& Fischer, H. (2011). Scaffolds for bone healing: concepts, materials and evidence.Injury, 42 (6), 569-573. doi:10.1016/j.injury.2011.03.033

Liliensiek, S. J., Wood, J. A., Yong, J., Auerbach, R., Nealey, P. F., \& Murphy, C. J. (2010). Modulation of human vascular endothelial cell behaviors by nanotopographic cues. Biomaterials, 31 (20), 5418-5426. doi:10.1016/j.biomaterials.2010.03.045

Lim, K. T., Suh, J. D., Kim, J., Choung, P. H., \& Chung, J. H. (2011). Calcium phosphate bioceramics fabricated from extracted human teeth for tooth tissue engineering. J Biomed Mater Res B Appl Biomater, 99 (2), 399-411. doi:10.1002/jbm.b.31912 
Liu, Y., Luo, D., \& Wang, T. (2016). Hierarchical Structures of Bone and Bioinspired Bone Tissue Engineering. Small, 12 (34), 4611-4632. doi:10.1002/smll.201600626

McMahon, R. E., Wang, L., Skoracki, R., \& Mathur, A. B. (2013). Development of nanomaterials for bone repair and regeneration. J Biomed Mater Res B Appl Biomater, 101 (2), 387-397. doi:10.1002/jbm.b.32823

Meng, X., \& Deng, D. (2016). Trash to Treasure: Waste Eggshells Used as Reactor and Template for Synthesis of Co9S8 Nanorod Arrays on Carbon Fibers for Energy Storage. Chemistry of Materials, 28 (11), 3897-3904. doi:10.1021/acs.chemmater.6b01142

Park, S., Choi, K. S., Lee, D., Kim, D., Lim, K. T., Lee, K.-H., . . . Kim, J. (2016). Eggshell membrane: Review and impact on engineering.Biosystems Engineering, 151 , 446-463. doi:10.1016/j.biosystemseng.2016.10.014

Rakhmatia, Y. D., Ayukawa, Y., Atsuta, I., Furuhashi, A., \& Koyano, K. (2015). Fibroblast attachment onto novel titanium mesh membranes for guided bone regeneration. Odontology, 103 (2), 218-226. doi:10.1007/s10266-014-0151-8

Rakhmatia, Y. D., Ayukawa, Y., Furuhashi, A., \& Koyano, K. (2013). Current barrier membranes: titanium mesh and other membranes for guided bone regeneration in dental applications. J Prosthodont Res, 57 (1), 3-14. doi:10.1016/j.jpor.2012.12.001

Sah, M. K., \& Pramanik, K. (2014). Soluble-eggshell-membrane-protein-modified porous silk fibroin scaffolds with enhanced cell adhesion and proliferation properties. Journal of Applied Polymer Science, 131 (8), 40138-40149. doi:10.1002/app.40138

Sah, M. K., \& Rath, S. N. (2016). Soluble eggshell membrane: A natural protein to improve the properties of biomaterials used for tissue engineering applications. Mater Sci Eng C Mater Biol Appl, 67 , 807-821. doi:10.1016/j.msec.2016.05.005

Sheikh, Z., Qureshi, J., Alshahrani, A. M., Nassar, H., Ikeda, Y., Glogauer, M., \& Ganss, B. (2017). Collagen based barrier membranes for periodontal guided bone regeneration applications. Odontology, 105 (1), 1-12. doi:10.1007/s10266-016-0267-0

Shim, J. H., Huh, J. B., Park, J. Y., Jeon, Y. C., Kang, S. S., Kim, J. Y., . . . Cho, D. W. (2013). Fabrication of blended polycaprolactone/poly(lactic-co-glycolic acid)/beta-tricalcium phosphate thin membrane using solid freeform fabrication technology for guided bone regeneration. Tissue Eng Part A, 19 (3-4), 317-328. doi:10.1089/ten.TEA.2011.0730

Shim, J. H., Yoon, M. C., Jeong, C. M., Jang, J., Jeong, S. I., Cho, D. W., \& Huh, J. B. (2014). Efficacy of rhBMP-2 loaded PCL/PLGA/beta-TCP guided bone regeneration membrane fabricated by 3D printing technology for reconstruction of calvaria defects in rabbit. Biomed Mater, 9 (6), 065006. doi:10.1088/1748$6041 / 9 / 6 / 065006$

Song, J. H., Kim, H. E., \& Kim, H. W. (2007). Collagen-apatite nanocomposite membranes for guided bone regeneration. J Biomed Mater Res B Appl Biomater, 83 (1), 248-257. doi:10.1002/jbm.b.30790

T. Viana, S. B., H. A. Almeida, P. J. Bartolo. (2014). PCL/EGGSHELL SCAFFOLDS FOR BONE REGENERATION. Proceedings of the ASME 2014 12th Biennial Conference on Engineering Systems Design and Analysis, 1 .

Teixeira, A. I., Abrams, G. A., Bertics, P. J., Murphy, C. J., \& Nealey, P. F. (2003). Epithelial contact guidance on well-defined micro-and nanostructured substrates. Journal of Cell Science, 116 (10), 1881-1892.

Versaevel, M., Grevesse, T., \& Gabriele, S. (2012). Spatial coordination between cell and nuclear shape within micropatterned endothelial cells. Nat Commun, 3 , 671. doi:10.1038/ncomms1668

Wang, Y., Kim, H. J., Vunjak-Novakovic, G., \& Kaplan, D. L. (2006). Stem cell-based tissue engineering with silk biomaterials.Biomaterials, 27 (36), 6064-6082. doi:10.1016/j.biomaterials.2006.07.008 
Yi, F., Guo, Z. X., Zhang, L. X., Yu, J., \& Li, Q. (2004). Soluble eggshell membrane protein: preparation, characterization and biocompatibility. Biomaterials, 25 (19), 4591-4599. doi:10.1016/j.biomaterials.2003.11.052

Yi, F., Yu, J., Guo, Z.-X., Zhang, L.-X., \& Li, Q. (2003). Natural Bioactive Material: A Preparation of Soluble Eggshell Membrane Protein.Macromolecular Bioscience, 3 , 234-237.

Zhang, E., Zhu, C., Yang, J., Sun, H., Zhang, X., Li, S., . . . Yao, F. (2016). Electrospun PDLLA/PLGA composite membranes for potential application in guided tissue regeneration. Mater Sci Eng C Mater Biol Appl, 58 , 278-285. doi:10.1016/j.msec.2015.08.032

Figure regends

Figure 1 . Fabrication and characteristic of SEP-coated PCL flat patch. (a) Schematic of the fabrication method for the SEP-coated PCL flat patch, including lithography and dip-coating, and optical image of a SEP-coated flexible PCL flat patch. (b) SEM images of the uncoated (Flat) and SEP-coated (E-flat) surfaces of the flat morphology. (c) AFM images of the uncoated and SEP-coated surfaces of the flat morphology (d) FT-IR analysis of the SEP, PCL flat patch, and SEP-coated PCL flat patch. (e) Contact angle measurements of the PCL and SEP-coated PCL flat patches. (f) Strain-stress curves of the PCL and SEP-coated PCL flat patches. (g) Adhesion force analysis of the PCL and SEP-coated PCL flat patch (left : normal adhesion, right: shear adhesion).

Figure 2. Effect of SEP-coated flat topography on cell morphology, proliferation, and differentiation. (a) Immunofluorescence image at the multi- and single-cell levels of phalloidin (red), vinculin (green), and DAPI (blue) of osteoblasts cultured on the SEP-coated flat topography. (b) Quantitative analysis of the cell body and nucleus (c) Correlation of cell the body with nucleus. (d) Number and size of focal adhesions on the SEPcoated flat topography. (e) Proliferation of cells on the SEP-coated flat topography. (f) Alkaline phosphatase staining of osteoblasts during osteogenesis induction on the SEP-coated flat topography. (g) Mineralization (ARS) staining of osteoblasts during osteogenesis on the SEP-coated flat topography.

Figure 3 . Fabrication and characteristics of the SEP-coated PCL nanopatch. (a) Schematic for the fabrication of the SEP-coated PCL nanopatch, including lithography and dip-coating, and optical image of a SEP-coated flexible PCL nanopatch. (b) SEM images of uncoated (Nano) and SEP-coated (E-nano) surfaces of the nano morphology. (c) AFM images of uncoated and SEP-coated surfaces of the nano morphology. (d) FT-IR analysis of the SEP, PCL nanopatch, and SEP-coated PCL nanopatch. (e) Contact angle measurements of the PCL and SEP-coated PCL nanopatches. (f) Tensile stress analysis of the PCL and SEP-coated PCL nanopatches. (g) Adhesion force analysis of the PCL and SEP-coated PCL nanopatches (left: normal adhesion, right: shear adhesion).

Figure 4. Effect of SEP-coated nanotopography on cell morphology, proliferation, and differentiation. (a) Immunofluorescence images at the multi- and single-cell levels of phalloidin (red), vinculin (green), and DAPI (blue) of osteoblasts cultured on the SEP-coated nanotopography. (b) Quantitative analysis of the cell body and nucleus (c) Correlation of the cell body and nucleus. (d) Proliferation of cells on the SEPcoated nanotopography. (e) Alkaline phosphatase staining of osteoblasts during osteogenesis induction on the SEP-coated nanotopography. (f) Mineralization staining of osteoblasts by osteogenesis on the SEP-coated nanotopography.

Figure 5 . Effect of SEP coating and nanotopographical cuesin vivo . (a) Representative micro-CT image, hematoxylin and eosin (H\&E) staining image, and (b) quantitative analysis of micro-CT of the bone regeneration of SEP-coated flat and nanotopography patches after 3 weeks of repair ( $\mathrm{n}=5$ per sample). (c) In vitro cell migration on nanotopographical and SEP-coated nanotopographical patches. (d) Quantification of cell migration distance, velocity, and covered area. (e) Western blot analysis and quantification of the expression levels of FAK and ERK of osteoblasts cultured on the SEP-coated flat topography for 2 days. (f) Schematic model of SEP coating and nanotopographical cue effects. The relative adhesion factor was obtained from the cell spreading ratio, adhesion force, and cell differentiation factor. 

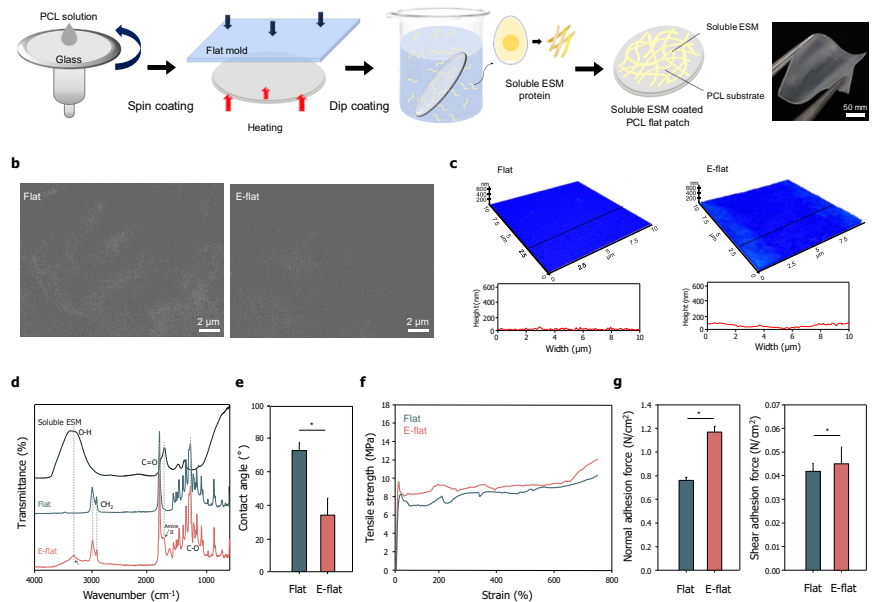

Figure 1. Fabrication and characteristic of SEP-coated PCL flat patch. (a) Schematic of the fabrication method for the SEP-coated PCL flat patch, including lithography and dip-coating, and optical image of a SEP-coated flexible PCL flat patch. (b) SEM images of the uncoated (Flat) and SEP-coated (E-flat) surfaces of the flat morphology. (c) AFM images of the uncoated and SEPcoated surfaces of the flat morphology (d) FT-IR analysis of the SEP, PCL flat patch, and SEPcoated PCL flat patch. (e) Contact angle measurements of the PCL and SEP-coated PCL flat patches. (f) Strain-stress curves of the PCL and SEP-coated PCL flat patches. (g) Adhesion force analysis of the PCL and SEP-coated PCL flat patch (left : normal adhesion, right: shear adhesion). 

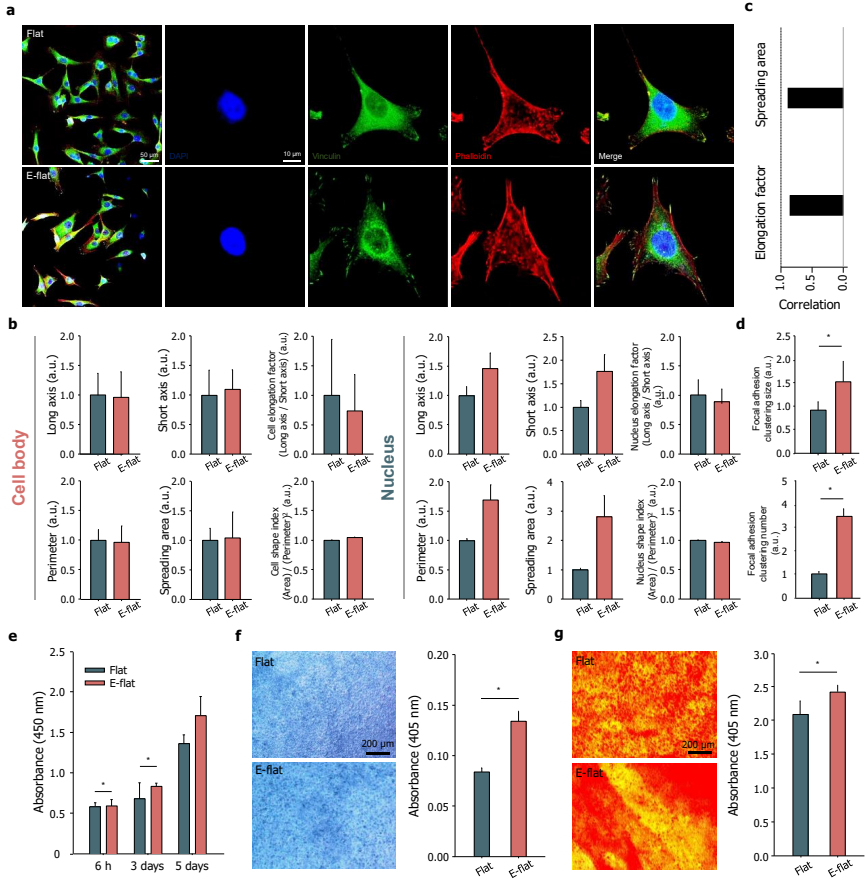

Figure 2. Effect of SEP-coated flat topography on cell morphology, proliferation, and differentiation. (a) Immunofluorescence image at the multi- and single-cell levels of phalloidin (red), vinculin (green), and DAPI (blue) of osteoblasts cultured on the SEP-coated flat topography. (b) Quantitative analysis of the cell body and nucleus (c) Correlation of cell the body with nucleus (d) Number and size of focal adhesions on the SEP-coated flat topography. (e) Proliferation of cells on the SEP-coated flat topography. (f) Alkaline phosphatase staining of osteoblasts during osteogenesis induction on the SEP-coated flat topography. (g) Mineralization (ARS) staining of osteoblasts during osteogenesis on the SEP-coated flat topography. 
a
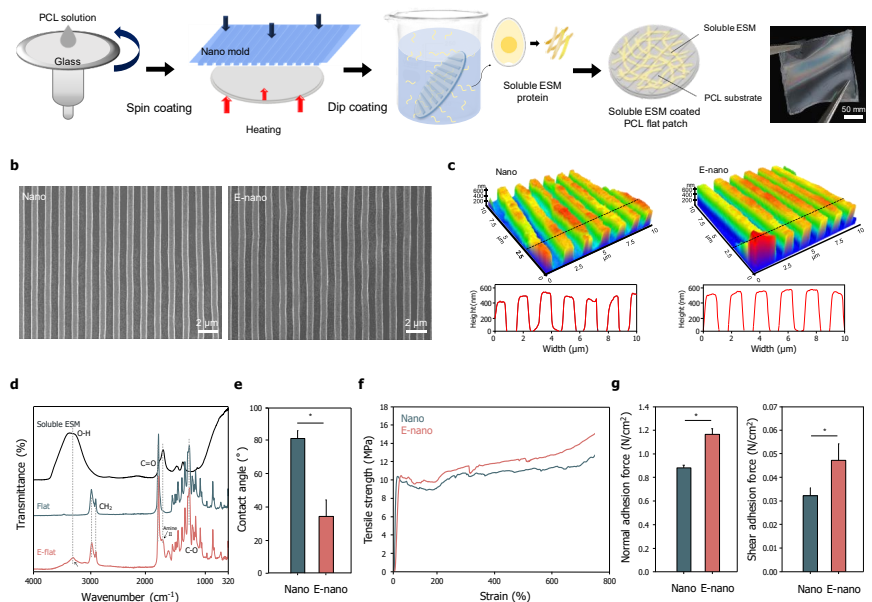

Figure 3. Fabrication and characteristics of the SEP-coated PCL nanopatch. (a) Schematic for the fabrication of the SEP-coated PCL nanopatch, including lithography and dip-coating, and optical image of a SEP-coated flexible PCL nanopatch. (b) SEM images of uncoated (Nano) and SEPcoated (E-nano) surfaces of the nano morphology. (c) AFM images of uncoated and SEP-coated surfaces of the nano morphology. (d) FT-IR analysis of the SEP, PCL nanopatch, and SEP-coated

PCL nanopatch. (e) Contact angle measurements of the PCL and SEP-coated PCL nanopatches. (f) Tensile stress analysis of the PCL and SEP-coated PCL nanopatches. (g) Adhesion force analysis of the PCL and SEP-coated PCL nanopatches (left: normal adhesion, right: shear adhesion). 


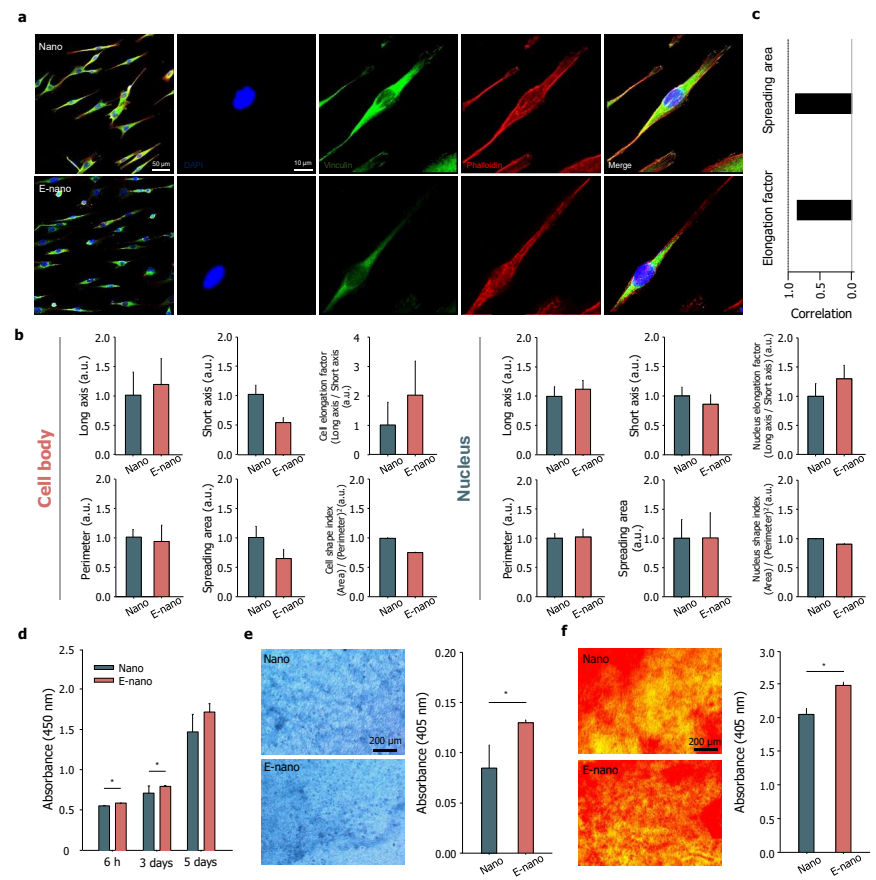

Figure 4. Effect of SEP-coated nanotopography on cell morphology, proliferation, and differentiation. (a) Immunofluorescence images at the multi- and single-cell levels of phalloidin (red), vinculin (green), and DAPI (blue) of osteoblasts cultured on the SEP-coated nanotopography. (b) Quantitative analysis of the cell body and nucleus (c) Correlation of the cell body and nucleus. (d) Proliferation of cells on the SEP-coated nanotopography. (e) Alkaline phosphatase staining of osteoblasts during osteogenesis induction on the SEP-coated nanotopography. (f) Mineralization staining of osteoblasts by osteogenesis on the SEP-coated nanotopography. 




Figure 5. Effect of SEP coating and nanotopographical cues in vivo. (a) Representative micro-CT image, hematoxylin and eosin (H\&E) staining image, and (b) quantitative analysis of micro-CT of the bone regeneration of SEP-coated flat and nanotopography patches after 3 weeks of repair $(n=$ 5 per sample). (c) In vitro cell migration on nanotopographical and SEP-coated nanotopographical patches. (d) Quantification of cell migration distance, velocity, and covered area. (e) Western blot analysis and quantification of the expression levels of FAK and ERK of osteoblasts cultured on the SEP-coated flat topography for 2 days. (f) Schematic model of SEP coating and nanotopographical cue effects. The relative adhesion factor was obtained from the cell spreading ratio, adhesion force, and cell differentiation factor. 